\title{
Surgical treatment of a retroperitoneal benign tumor surrounding important blood vessels by fractionated resection: A case report and review of the literature
}

\author{
ZHILI WAN, TIANSHENG YIN, HONGWEI CHEN and DEWEI LI \\ Department of Hepatobiliary Surgery, The First Affiliated Hospital of Chongqing Medical University, \\ Chongqing 400016, P.R. China
}

Received March 5, 2015; Accepted December 10, 2015

DOI: $10.3892 / \mathrm{ol} .2016 .4395$

\begin{abstract}
Retroperitoneal tumors are lesions with diverse pathological subtypes that originate from the retroperitoneal space; $\sim 40 \%$ of these tumors are benign. Due to such lesions often surrounding and associating with vital abdominal blood vessels, a complete surgical resection is difficult. The current study presents a novel surgical approach, known as fractionation, through which a benign retroperitoneal tumor surrounding important abdominal blood vessels was completely resected. A 21-year-old man was admitted to The First Affiliated Hospital of Chongqing Medical University (Chongqing, China), presenting with a $\sim 7.5 \times 7.2-\mathrm{cm}$ tumor that was located in the retroperitoneal pancreatic head region and the first hepatic hilum. The tumor completely surrounded the celiac axis and the splenic, common hepatic and superior mesenteric arteries, and was closely associated with the abdominal aorta and the portal, splenic, superior mesenteric and left renal veins. A pre-operative computed tomography scan and intraoperative frozen biopsy indicated that the lesion was a benign tumor. A fractionation approach was subsequently adopted, with fractionation of the lesion being performed according to the location of the tumor itself and the direction of the surrounding abdominal blood vessels. In this manner, a complete tumor resection was conducted. Post-operative pathological examination confirmed the diagnosis of a retroperitoneal ganglioneuroma. The patient was followed up for a year and a half, with no evidence of tumor recurrence. In the present case, a fractionation approach for the complete resection of the retroperitoneal benign tumor
\end{abstract}

Correspondence to: Professor Dewei Li, Department of Hepatobiliary Surgery, The First Affiliated Hospital of Chongqing Medical University, 1 Friendship Road, Chongqing 400016, P.R. China

E-mail: lidewei406@sina.com

Key words: retroperitoneal benign tumor, vascular involvement, fractionated resection, vascular protection achieved a positive outcome and demonstrated the feasibility of the technique.

\section{Introduction}

Retroperitoneal tumors are rare lesions with diverse pathological subtypes, which originate from the retroperitoneal space (1). In total, $40 \%$ of retroperitoneal tumors are benign (2). Benign retroperitoneal tumors are slow-growing and typically lack a specific clinical manifestation. The primary characteristic of this lesion is the compression of the tumor surroundings, therefore, early diagnosis is often difficult (3). Pre-operative diagnosis predominantly relies on imaging techniques, including enhanced computed tomography (CT) and magnetic resonance imaging (4). Retroperitoneal tumors often surround and associate with vital abdominal organs and blood vessels, thus, performing a complete surgical resection is challenging (5). Nevertheless, follow-up data from previous studies have demonstrated that patients who have undergone a complete resection have significantly higher 1-, 3- and 5-year survival rates than those who have undergone an incomplete resection. Therefore, the resection rate significantly impacts the post-operative local recurrence rate and prognosis (6). Even if a retroperitoneal tumor is diagnosed as benign, if it is not removed completely then the tumor may result in the compression of abdominal organs, tissues, blood vessels and nerves, followed by continual tumor growth and possible fatality (7). Certain researchers consider the invasion of benign retroperitoneal tumors into the surrounding abdominal blood vessels and organs to be an independent limiting factor for their complete surgical resection (8). Given that such lesions are more likely to surround, rather than infiltrate, abdominal vascular walls, the present study designed a novel surgical approach, known as the fractionation approach. This approach resulted in the fractionation and subsequent complete resection of a giant benign retroperitoneal tumor that surrounded major blood vessels, including the celiac axis and the splenic, common hepatic and superior mesenteric arteries, and was closely associated with the abdominal aorta and the portal, splenic, superior mesenteric and left renal veins. During this technique, the important retroperitoneal blood vessels were protected effectively. 


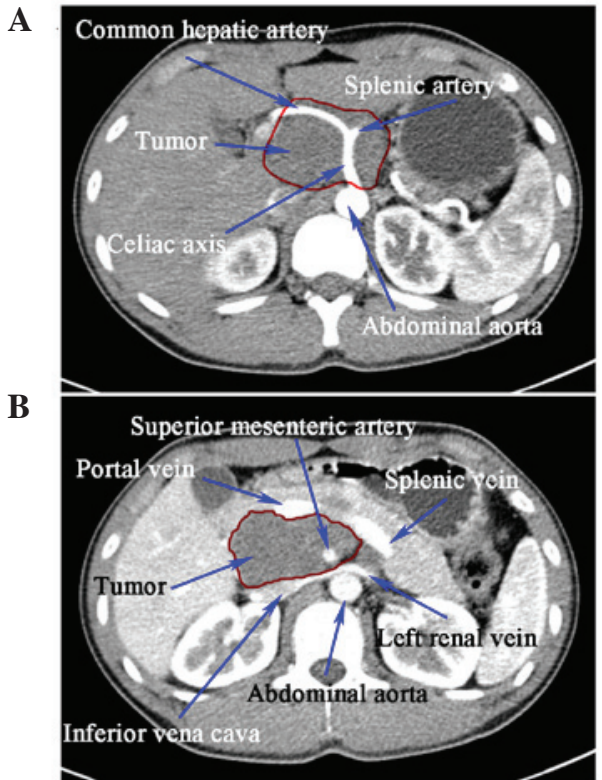

Figure 1. Enhanced computed tomography scan demonstrating the tumor located at the rear of the pancreas and near the hepatic portal, with unclear boundaries at the pancreatic head. (A) The tumor completely surrounds the celiac axis and the common hepatic and splenic arteries, and is also adjacent to the abdominal aorta. (B) The tumor fully surrounds the superior mesenteric artery, and is closely associated with the portal, splenic and left renal veins, and the pancreatic head. The red lines indicate the boundary of the tumor.

\section{Case report}

A 21-year-old man was admitted to The First Affiliated Hospital of Chongqing Medical University (Chongqing, China) in March 2013 presenting with a mass in the pancreatic head region that had been identified during a physical examination 9 months previously. The patient also complained of subxiphoid discomfort for $>1$ month. A total of 9 months prior to admission, the patient had undergone abdominal ultrasonography at another hospital, which resulted in the detection of a mass measuring $\sim 6.5 \times 4.5 \mathrm{~cm}$, located in the pancreatic head region and the first hepatic hilum. As the mass was asymptomatic, the patient did not undergo treatment. Furthermore, 1 month prior to admission, paroxysmal pain and discomfort was experienced below the xiphoid, and abdominal enhanced CT (SOMATOM ${ }^{\circledR}$ Definition Flash Dual Source CT Scanner; Siemens AG, Munich, Germany) identified a mass $\sim 7.2 \times 4.3 \times 7.5 \mathrm{~cm}$ in size located in the rear of the pancreatic head and body. The tumor completely surrounded the celiac axis and the splenic, common hepatic and superior mesenteric arteries, and was closely associated with the abdominal aorta and the portal, splenic, superior mesenteric and left renal veins. Additionally, the mass invaded the pancreatic head and compressed the pancreatic body (Fig. 1). Pre-operative $\mathrm{CT}$ also indicated the presence of a benign tumor, which was diagnosed as benign since the tumor had a clear margin, smooth surface, symmetrical density and a blood supply, the lymph nodes surrounding the tumor were not enlarged and the abdominal blood vessels did not have a filling defect. An intraoperative frozen biopsy confirmed the diagnosis of a benign tumor. Subsequently, a retroperitoneal tumor resection and a pancreaticoduodenectomy were performed.
A

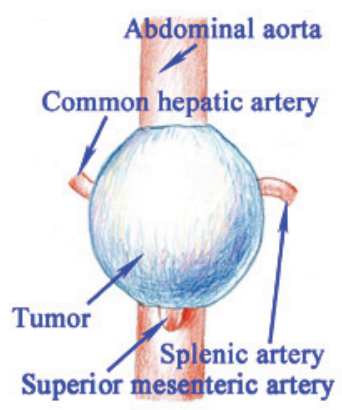

B

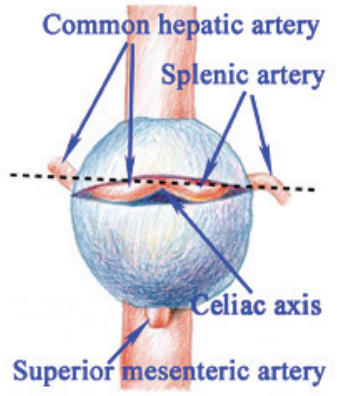

C



D

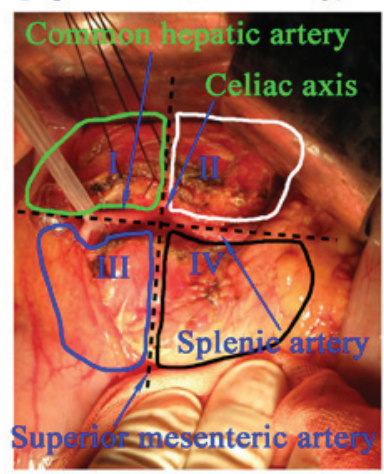

Figure 2. Fractionation procedure. (A) The tumor completely envelops the celiac axis and the initial segments of the common hepatic, splenic and superior mesenteric arteries. (B) Common hepatic and splenic arteries are identified, which are used as guidelines to make a straight line. The tumor is incised along this line and divided into two parts, with the celiac axis now exposed. A straight line is made between the celiac axis and superior mesenteric artery, dividing the tumor into two fractions. (C) Thus, the tumor is now divided into four quadrants as follows: The upper right (II), upper left (I), lower right (IV) and lower left (III). (D) Fractions I, II, III and IV in real images; such tumors are divided into four fractions intraoperatively.

The tumor was exposed during surgery and $0.6 \times 0.6 \mathrm{~cm}$ of tissue was removed as an intraoperative frozen biopsy sample, which was later established to be benign. The association between the tumor and its surrounding structures was analyzed. It was observed that the lesion completely surrounded the celiac axis and the splenic, common hepatic and superior mesenteric arteries, and was closely associated with the splenic, portal and left renal veins, and the abdominal aorta (Fig. 2A). The 


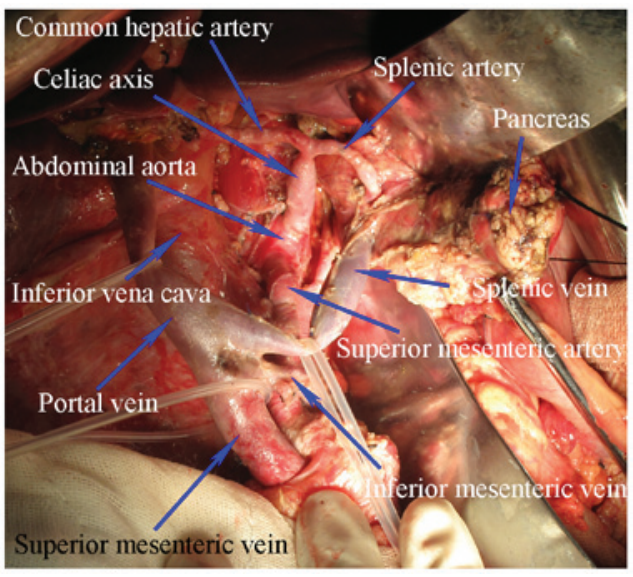

Figure 3. Image captured following completion of surgery. The locations of the following structures following tumor resection can be clearly identified: The portal, splenic, superior and inferior mesenteric veins, the celiac axis, the common hepatic artery and the pancreatic stump. Following removal of the tumor, the important abdominal blood vessels remained intact.

\section{A}

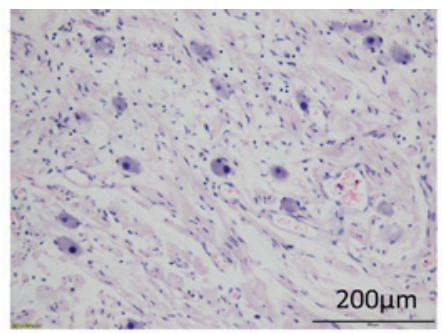

B

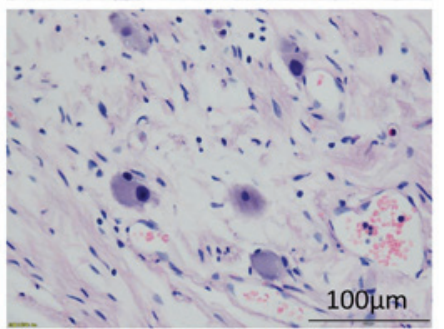

C

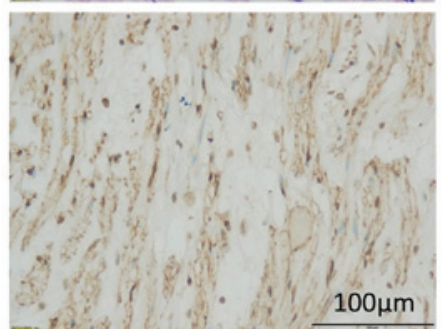

D

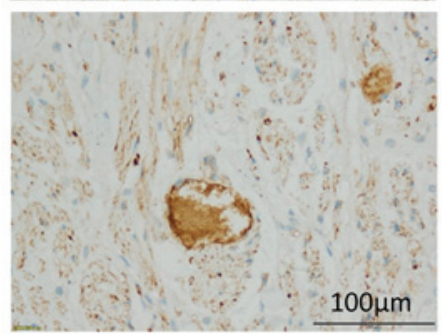

Figure 4. (A and B) Mature ganglion cells observed within the tumor using hematoxylin and eosin staining. Immunohistochemical staining of ganglion cells that reacted strongly with (C) S-100 and (D) synaptophysin. (stain, hematoxylin and eosin; magnification, $\mathrm{x} 40$ ).

lesion, however, could not be separated from the head of the pancreas. Therefore, a fractionated resection of the tumor was
A

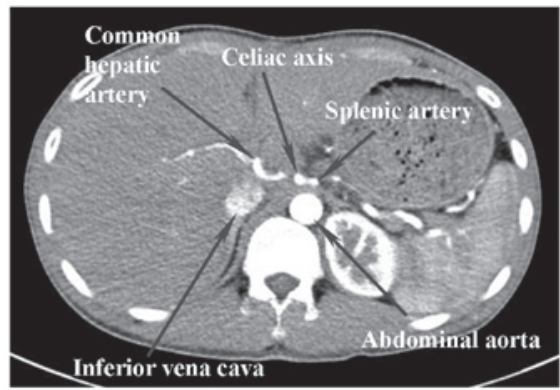

B

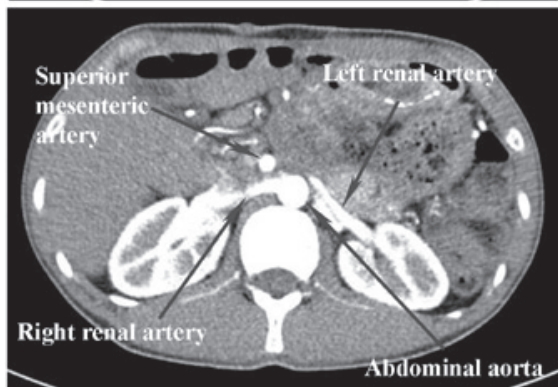

C

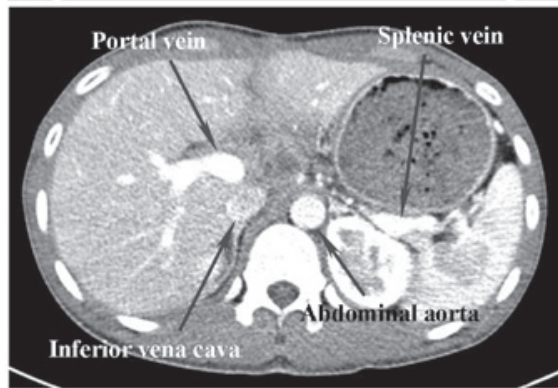

D

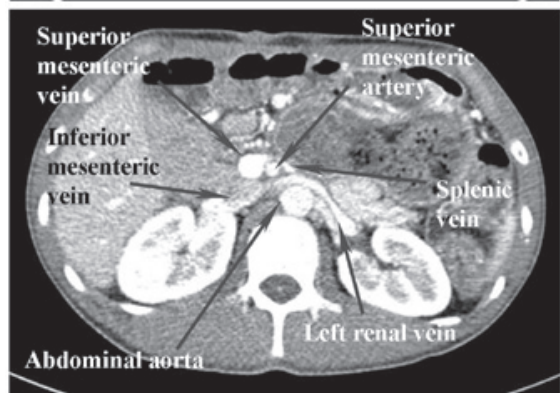

Figure 5. Abdominal computed tomography rescan at 1 year post-surgery showing no tumor recurrence. (A) The common hepatic and splenic arteries, and the celiac axis are intact. (B) The abdominal aorta and superior mesenteric artery. (C) The portal and splenic veins and the inferior vena cava are patent. (D) The splenic and superior mesenteric veins, and the inferior vena cava are patent.

performed, as well as a pancreaticoduodenectomy. The gastric antrum and the pancreatic neck were severed, and the splenic, portal and superior and inferior mesenteric veins were isolated. The tumor, which was located behind the pancreas and the aforementioned veins, was now fully exposed. Bounded by the splenic and common hepatic arteries, the tumor was divided into upper and lower fractions (Fig. 2B). The tumor was incised according to the courses of the common hepatic and splenic arteries, which were then exposed to the celiac axis, and the left gastric artery was severed. Also bounded by the celiac axis and the superior mesenteric artery, the tumor mass was further divided into left and right fractions (Fig. 2C). The tumor was incised according to the courses of the celiac axis and the superior mesenteric artery. Subsequently, the celiac axis and 
superior mesenteric artery were exposed to the surface of the abdominal aorta. The tumor fractions were resected separately in the following sequence: Upper right, lower right, upper left and lower left (Fig. 2C and D). The important blood vessels were protected sufficiently (Fig. 3). The remaining pancreaticoduodenectomy steps were performed in a routine manner.

Pathological analysis identified that the neoplasm was composed of numerous scattered, mature ganglion cells (Fig. 4A and B). No association was observed at the junction of the pancreas and intestine, or near the resected margin of the pancreas and intestine. Immunohistochemical staining exhibited a positive S100 reaction in the membrane of the ganglion cells fibers (Fig. 4C), whereas the cytoplasm of the ganglion cells reacted strongly with synaptophysin (Fig. 4D). Immunohistochemistry was performed by the Clinical Pathological Diagnosis Center of Chongqing Medical University (Chongqing, China).

The patient was followed up for a year and a half, and appeared to be in a good condition without discomfort. An abdominal CT scan demonstrated no tumor recurrence, whilst the celiac axis and splenic, common hepatic and superior mesenteric arteries, and the abdominal aorta were intact, and the superior mesenteric and portal veins were patent (Fig. 5).

Written informed consent was obtained from the patient prior to the publication of the present study.

\section{Discussion}

Retroperitoneal tumors are lesions that originate from the retroperitoneal space, presenting with diverse pathological subtypes $(1,9)$. Of all retroperitoneal tumors, benign tumors account for $\sim 40 \%(2,10)$. Benign retroperitoneal tumors have a wide range with regard to age of onset, with the lesions typically growing slowly and exhibiting no symptoms at the early stages (9,11-13). Patients frequently seek treatment due to experiencing chronic abdominal pain and bloating, resulting from compression of the surrounding tissues by larger tumors $(9,14)$. By the time a diagnosis is reached, tumors may have already surrounded and invaded important abdominal blood vessels, subsequently making a complete surgical resection difficult (5). Furthermore, surgery may easily lead to the injury of vital organs and blood vessels, as well as multiple post-operative complications. Nevertheless, if benign tumors are resected incompletely, post-operative recurrence and tumor regrowth may lead to a similar outcome as observed with malignancies, and may ultimately lead to fatality (7). Therefore, the search for a surgical approach that allows complete resection of a tumor, whilst protecting important blood vessels, tissues and organs, appears to be necessary.

Benign retroperitoneal tumors are slow-growing and non-invasive (12). Such lesions typically surround and compress major blood vessels close to the abdominal cavity, rather than directly infiltrating or damaging the vascular walls, as observed in malignancies. Furthermore, in benign retroperitoneal tumors, there is typically little concern regarding tumor implantation and metastasis resulting from tumor rupture during surgical resection. Thus, the implementation of a complete resection of these benign tumors is made possible using the fractionation approach, according to the orientation of the major retroperitoneal blood vessels, which surround important abdominal blood vessels.

The present study reviewed the literature of the recent decades regarding the surgical treatment of retroperitoneal tumors. Only a small number of studies reported and discussed this method for the resection of such lesions $(5,13)$. Certain studies have described cases of laparoscopic resection of retroperitoneal tumors. Ahn et al (15) described a laparoscopic approach for the resection of retroperitoneal tumors, which had the advantages of minimal damage to surrounding tissues, reduced bleeding and fewer complications compared with traditional open dissection (13,15-17). However, in the case of larger tumors, severe intraperitoneal adhesions or hemorrhages, laparoscopic surgery may face limitations, particularly when treating retroperitoneal tumors that surround and invade major blood vessels within the abdominal cavity $(3,17)$. Subsequently, a traditional open dissection becomes necessary.

Kariya et al (18) described the use of radiofrequency ablation followed by injections of carbon dioxide $\left(\mathrm{CO}_{2}\right)$ between the retroperitoneal tumor capsule and the adjacent tissues and organs. The principle of such injections is that $\mathrm{CO}_{2}$ serves an important role in thermal isolation, which may prevent thermal injury to the surrounding tissues during radiofrequency ablation. However, this method does present with limitations when the tumors are surrounding important abdominal blood vessels, particularly multiple abdominal vessels at numerous locations. Furthermore, a percutaneous injection of $\mathrm{CO}_{2}$ is difficult to execute, and has the potential to cause heavy bleeding or air embolization. $\mathrm{CO}_{2}$ embolization, caused by laparoscopic surgery, has been previously reported (19). Therefore, the effectiveness of the surgical procedure remains controversial.

Regarding retroperitoneal tumors that invade important abdominal blood vessels, the literature describes a different treatment using combined major vascular resection. For example, Fueglistaler et al (8) noted a combined approach using a partial vascular resection and revascularization for the treatment of retroperitoneal tumors that had invaded the abdominal blood vessels. However, this approach has its own limitations, including a risk of heavy bleeding during vascular resection and prosthetic vessel replacement. Intraoperative arterial occlusion may result in ischemia and hypoxia in corresponding tissues and organs, whilst venous occlusion may result in distal tissue and organ congestion. Multiple studies have demonstrated that if hepatic vascular occlusion is experienced for $>60 \mathrm{~min}$, this contributes to the risk of post-operative liver failure $(11,20)$. If complete renal artery occlusion is encountered for a period of $30 \mathrm{~min}$, this may affect the post-operative renal function of patients (11,21-25). Prolonged renal artery occlusion may cause renal failure, pale hypertension and kidney necrosis, whilst mesenteric vessel occlusion may cause bowel necrosis. Regarding vascular replacement-associated complications, the incidence of early complications following vena cava replacement is $\sim 44 \%$, which includes intraoperative coagulation disorders, prosthetic clogging and prosthetic infection, possibly leading to fatality (8). In the study by Fueglistaler et al (8), it was reported that the early complication rate subsequent to aorta and vena cava replacement, performed using different vascular replacement approaches, remained at $19 \%$. Therefore, combined vascular resection and replacement approaches have their drawbacks. Nevertheless, 
such approaches are considered to be the only methods of treatment for advanced tumors, particularly malignant tumors that exhibit vascular invasion (8). The surgical approach for the treatment of benign retroperitoneal tumors presenting with vascular invasion should be successful in fully removing the tumors, whilst effectively protecting blood vessels; therefore, the surgical approach may significantly lower the incidence of post-operative complications and reduce the cost of surgery.

The treatment of tumors associated with retroperitoneal blood vessels, and even organs, using traditional methods may inflict greater risks and difficulties when conducting the surgery due to their vascular invasion. In particular, important retroperitoneal organs (including the liver, spleen, pancreas), and even blood vessels (such as the celiac artery and vein), may affect the safety of surgery when it is subsequent to injury, possibly leading to severe bleeding and decreased patient survival (26). Similarly, injury to specific blood vessels, including the renal artery, may cause uncontrolled hypertension (26) and an increasing risk of perioperative cardiocerebral events. Furthermore, this may lead to multiple post-operative complications and possibly affect the prognosis and quality of life of patients.

When considering the fractionated resection approach for tumors that surround blood vessels, identification of the status of important abdominal blood vessels that have been invaded by the tumor is required. Subsequently, the tumor should be divided into different areas, according to the blood vessels that surround the lesion and serve as guidelines. Finally, the tumor may be resected separately and completely. This method does not only expose and protect important abdominal blood vessels, lower the risk of severe bleeding caused by major abdominal vascular injury and reduce post-operative complications, but it is also conducive to a complete resection of the tumor. In the present case, the retroperitoneal tumor was $\sim 7.2 \times 4.3 \times 7.5 \mathrm{~cm}$ in size, completely surrounded the celiac axis and the common hepatic, splenic and superior mesenteric arteries, and was also closely associated with the abdominal aorta and portal, superior mesenteric, splenic and left renal veins. Complete surgical resection was, however, challenging. Pre-operative CT and the intraoperative frozen biopsy indicated the presence of a benign tumor. Using the fractionation approach, a straight line was made along the direction of the common hepatic and splenic arteries, which subsequently divided the tumor into upper and lower fractions. The tumor was then incised between the upper and lower fractions, and the celiac axis was gradually exposed according to the direction of the common hepatic and splenic arteries. Following this, the tumor was divided into left and right fractions along the direction of the celiac axis and the superior mesenteric artery. The tumor was then incised between the left and right fractions, and the abdominal aorta was gradually exposed whilst attention was paid to protect the celiac axis and superior mesenteric artery. At this point, the tumor was divided into four fractions, namely the upper right, lower right, upper left and lower left. The lesion was then resected separately whilst the important abdominal blood vessels (including the celiac axis, and the common hepatic, splenic and superior mesenteric arteries) were preserved (Fig. 2). Benign tumors that surround and invade blood vessels in other areas of the abdomen should also be able to be resected in a similar manner using the fractionation approach, whilst maximizing the protection of important blood vessels.

The fractionation approach is specifically designed for benign retroperitoneal tumors surrounding important abdominal blood vessels. Regarding retroperitoneal malignancies, fractionated resection runs the risk of tumor rupture, implantation and metastasis. Similarly, malignancies are prone to directly infiltrate and grow into the vascular walls, therefore limiting the likelihood of achieving radical resection. At this time, combination with vascular resection or replacement may be likely to improve the patient survival rate (5).

In conclusion, fractionated resection of benign retroperitoneal tumors may protect the invaded, surrounding blood vessels, whilst also achieving a complete radical resection. In the present case, no recurrence was observed during a year and a half of follow-up, with the invaded, surrounding blood vessels being preserved.

\section{Acknowledgements}

This study received a grant from the Chongqing Science and Technology Commission (grant no., cstc2012jjA0313).

\section{References}

1. Strauss DC, Hayes AJ and Thomas JM: Retroperitoneal tumours: Review of management. Ann R Coll Surg Engl 93: 275-280, 2011.

2. Nakashima J, Ueno M, Nakamura K, Tachibana M, Baba S, Deguchi N, Tazaki H and Murai M: Differential diagnosis of primary benign and malignant retroperitoneal tumors. Int J Urol 4: 441-446, 1997.

3. Hanaoka M, Hashimoto M, Sasaki K, Matsuda M, Fujii T, Ohashi K and Watanabe G: Retroperitoneal cavernous hemangioma resected by a pylorus preserving pancreaticoduodenectomy. World J Gastroenterol 19: 4624-4629, 2013.

4. Taskin HE and Berber E: Retroperitoneal tumors that may be confused as adrenal pathologies. J Surg Oncol 106: 600-603, 2012.

5. Tseng WW, Wang SC, Eichler CM, Warren RS and Nakakura EK: Complete and safe resection of challenging retroperitoneal tumors: Anticipation of multi-organ and major vascular resection and use of adjunct procedures. World J Surg Oncol 9: 143, 2011.

6. Xu YH, Guo KJ, Guo RX, Ge CL, Tian YL and He SG: Surgical management of 143 patients with adult primary retroperitoneal tumor. World J Gastroenterol 13: 2619-2621, 2007.

7. Nogami M, Hoshi T, Toukairin Y, Arai T, Uozaki H and Fukusato T: A case of the large retroperitoneal solitary fibrous tumor. Leg Med (Tokyo) 15: 19-22, 2013.

8. Fueglistaler P, Gurke L, Stierli P, Obeid T, Koella C, Oertli D and Kettelhack C: Major vascular resection and prosthetic replacement for retroperitoneal tumors. World J Surg 30: 1344-1349, 2006.

9. Virseda Rodríguez JA, Donate Moreno MJ, Pastor Navarro H, Carrión López P, Martínez Ruiz J, Martínez Sanchiz C and Perán Teruel M: Primary retroperitoneal tumors: Review of our 10-year case series. Arch Esp Urol 63: 13-22, 2010.

10. Egawa S, Satoh T, Suyama K, Uchida T, Iwabuchi K and Koshiba K: Giant retroperitoneal cyst in an adult male. Int J Urol 3: 304-306, 1996.

11. Shindo S, Motohashi S, Katsu M, Kaga S, Inoue H, Matsumoto M, Kono K, Fujii H and Takeda M: Treatment of abdominal malignancy invading the vena cava: A report of seven cases. Surg Today 37: 685-689, 2007.

12. Strauss DC, Qureshi YA, Hayes AJ and Thomas JM: Management of benign retroperitoneal schwannomas: A single-center experience. Am J Surg 202: 194-198, 2011.

13. Wei TC, Chung HJ, Lin AT and Chen KK: Robot-assisted laparoscopic excision of a retroperitoneal paracaval tumor. J Chin Med Assoc 76: 724-726, 2013.

14. Navin P, Meshkat B, McHugh S, Beegan C, Leen E, Prins H and Aly S: Primary retroperitoneal mucinous cystadenoma - A case study and review of the literature. Int J Surg Case Rep 3: 486-488, 2012. 
15. Ahn KS, Han HS, Yoon YS, Kim HH, Lee TS, Kang SB and Cho JY: Laparoscopic resection of nonadrenal retroperitoneal tumors. Arch Surg 146: 162-167, 2011.

16. Lehrfeld T, Natale R, Sharma S, Mendoza PJ, Schwab Li CW and Lee DI: Robot-assisted excision of a retroperitoneal mass between the left renal artery and vein. JSLS 14: 447-449, 2010.

17. Zhang Z and Xiu D: Laparoscopic surgery for primary retroperitoneal tumors: A single institution experience of 14 cases. Surg Laparosc Endosc Percutan Tech 20: 399-403, 2010.

18. Kariya S, Tanigawa N, Kojima H, Komemushi A, Shomura Y, Ueno Y, Shiraishi T and Sawada S: Radiofrequency ablation combined with $\mathrm{CO}_{2}$ injection for treatment of retroperitoneal tumor: Protecting surrounding organs against thermal injury. AJR Am J Roentgenol 185: 890-893, 2005.

19. Park EY, Kwon JY and Kim KJ: Carbon dioxide embolism during laparoscopic surgery. Yonsei Med J 53: 459-466, 2012.

20. Huguet C, Gavelli A, Chieco PA, Bona S, Harb J, Joseph JM, Jobard J, Gramaglia M and Lasserre M: Liver ischemia for hepatic resection: Where is the limit? Surgery 111: 251-259, 1992.

21. Kowalczyk KJ, Alemozaffar M, Hevelone ND, Ulmer WD, Plaster BA, Lipsitz SR, Yu HY and Hu JC: Partial clamping of the renal artery during robot-assisted laparoscopic partial nephrectomy: Technique and initial outcomes. J Endourol 26: 469-473, 2012.
22. Shao P, Qin C, Yin C, Meng X, Ju X, Li J, Lv Q, Zhang W and $\mathrm{Xu} Z$ : Laparoscopic partial nephrectomy with segmental renal artery clamping: Technique and clinical outcomes. Eur Urol 59: 849-855, 2011.

23. Shao P, Tang L, Li P, Xu Y, Qin C, Cao Q, Ju X, Meng X, Lv Q, $\mathrm{Li}$ J, et al: Application of a vasculature model and standardization of the renal hilar approach in laparoscopic partial nephrectomy for precise segmental artery clamping. Eur Urol 63: 1072-1081, 2013.

24. Gill IS, Eisenberg MS, Aron M, Berger A, Ukimura O, Patil MB, Campese V, Thangathurai D and Desai MM: 'Zero ischemia' partial nephrectomy: Novel laparoscopic and robotic technique. Eur Urol 59: 128-134, 2011

25. Gong EM, Zorn KC, Orvieto MA, Lucioni A, Msezane LP and Shalhav AL: Artery-only occlusion may provide superior renal preservation during laparoscopic partial nephrectomy. Urology 72: 843-846, 2008

26. Jones VS and Burns CR: Operative considerations in pediatric retroperitoneal teratomas - a review. Eur J Pediatr Surg 23: 265-269, 2013. 